\title{
OPTIMAL SOLUTIONS TO STOCHASTIC DIFFERENTIAL INCLUSIONS
}

Abstract. A martingale problem approach is used first to analyze compactness and continuous dependence of the solution set to stochastic differential inclusions of Ito type with convex integrands on the initial distributions. Next the problem of existence of optimal weak solutions to such inclusions and their dependence on the initial distributions is investigated.

1. Introduction. Stochastic inclusions appear in a natural way as a reduced or theoretical description of stochastic control problems (see e.g. [7] and references therein). The major contributions in this field concern strong solutions to stochastic inclusions. On the other hand M. Kisielewicz [8] investigated the main properties of weak solutions. The aim of this work is to study optimal weak solutions to such inclusions. We use the so called martingale problem approach owing to which some results obtained in [8] can be stated under weaker assumptions and in more suitable form for our aims.

Consider the stochastic differential inclusion

$$
\begin{aligned}
d \xi_{t} & \in F\left(t, \xi_{t}\right) d t+G\left(t, \xi_{t}\right) d W_{t}, \quad t \in[0, T], \\
P^{\xi_{0}} & =\mu,
\end{aligned}
$$

where $F:[0, T] \times \mathbb{R}^{d} \rightarrow \operatorname{Conv}\left(\mathbb{R}^{d}\right)$ and $G:[0, T] \times \mathbb{R}^{d} \rightarrow \operatorname{Conv}\left(\mathbb{R}^{d \times m}\right)$ are measurable, compact and convex valued multifunctions, $W$ is an $m$-dimensional Wiener process on the filtered probability space $(\Omega, \mathcal{F}$, $\left.\left\{\mathcal{F}_{t}\right\}_{0 \leq t \leq T}, P\right)$, and $\mu$ is a given probability measure on the space $\left(\mathbb{R}^{d}, \mathcal{B}\left(\mathbb{R}^{d}\right)\right)$. Here $\operatorname{Conv}()$ denotes the space of nonempty, convex and compact subsets of the underlying space. By $\mathbb{R}^{d \times m}$ we denote the space of all $d \times m$ ma-

2000 Mathematics Subject Classification: 93E03, 93C30.

Key words and phrases: stochastic differential inclusions, weak solutions, martingale problem, weak convergence of probability measures. 
trices $\left(g_{i j}\right)_{d \times k}$ with real elements, equipped with the norm $\left\|\left(g_{i j}\right)_{d \times m}\right\|=$ $\max _{1 \leq i \leq d, 1 \leq j \leq m}\left|g_{i j}\right|$.

For an $\mathbb{R}^{d}$-valued stochastic process $X$, let $X_{t}^{*}=\sup _{0 \leq s \leq t}\left|X_{s}\right|$. We denote by $\mathcal{F}_{t}^{X}=\sigma\left\{X_{s} ; s \leq t\right\}$ the "history of $X$ " ( $\sigma$-field generated by $\left.X\right)$ until time $t$. Finally, $E_{P}$ denotes expectation with respect to the probability $P$.

The basic notion in the paper is that of a weak solution:

Definition 1. By a weak solution to the stochastic inclusion (1) we mean a $d$-dimensional, continuous stochastic process $\xi$ defined on the probability space $(\Omega, \mathcal{F}, P)$ together with, a Wiener process $\left(W_{t}, \mathcal{F}_{t}^{\xi}\right)$, and $\left(\mathcal{F}_{t}^{\xi}\right)$ adapted processes $f_{t} \in F\left(t, \xi_{t}\right), g_{t} \in G\left(t, \xi_{t}\right) d t \times d P$-a.e. such that:

$$
\begin{aligned}
\xi_{t} & =\xi_{0}+\int_{0}^{t} f_{s} d s+\int_{0}^{t} g_{s} d W_{s}, \quad t \in[0, T], \\
P^{\xi_{0}} & =\mu,
\end{aligned}
$$

provided the above integrals exist.

We denote such a solution by $\left(\Omega, \mathcal{F}, P, W_{t},\left(\mathcal{F}_{t}^{\xi}\right)_{t \in[0, T]}, \xi\right)$.

Remark 1. Note that in the single-valued case this notion coincides with that of a weak solution to a stochastic equation, which can be found in $[3,5,13,14]$.

In [9] the problem of existence of weak solutions to the stochastic inclusion

$$
\begin{aligned}
d \xi_{t} & \in F\left(t, \xi_{t}\right) d t+g\left(t, \xi_{t}\right) d W_{t}, \quad t \geq 0, \\
P^{\xi_{0}} & =\mu,
\end{aligned}
$$

was considered with the set-valued $\operatorname{drift} F$ being bounded and jointly upper semicontinuous (u.s.c.), and with a single-valued diffusion term, i.e. a continuous bounded function $g: \mathbb{R}_{+} \times \mathbb{R}^{d} \rightarrow \mathbb{R}^{d \times m}$. The case of inclusions with set-valued drift and diffusion terms being jointly upper semicontinuous was investigated by J. P. Aubin and G. Da Prato [1].

Another type of assumptions on set-valued integrands involves lower semicontinuity (1.s.c.) with respect to the second variable (see [8]). For the notions of continuity, upper and lower semicontinuity, and measurability of set-valued mappings we refer to [4] or [6]. Let us close the introduction with the following result needed in what follows.

Theorem 1 (Kisielewicz [8]). Let $F:[0, T] \times \mathbb{R}^{d} \rightarrow \operatorname{Conv}\left(\mathbb{R}^{d}\right)$ and $G:$ $[0, T] \times \mathbb{R}^{d} \rightarrow \operatorname{Conv}\left(\mathbb{R}^{d \times m}\right)$ be measurable bounded set-valued mappings such that $F(t, \cdot)$ and $G(t, \cdot)$ are l.s.c. for fixed $t \in[0, T]$. Let $\mu$ be a probability measure on $\left(\mathbb{R}^{d}, \mathcal{B}\left(\mathbb{R}^{d}\right)\right)$. Then there exists a weak solution to the stochastic inclusion (1). 
The existence result for the inclusion (1) is a starting point to considering the optimality problem. In Section 4, we shall consider conditions under which there exist optimal weak solutions to (1), i.e. solutions $\xi^{*}$ satisfying

$$
E \int_{0}^{T} h\left(t, \xi_{t}^{*}\right) d t=\sup _{\xi} E \int_{0}^{T} h\left(t, \xi_{t}\right) d t
$$

for a given bounded continuous function $h:[0, T] \times \mathbb{R}^{d} \rightarrow \mathbb{R}$, where the supremum is taken over the set of all weak solutions to (1).

2. Weak solutions and a local martingale problem. Let $(\Omega, \mathcal{F}, P$, $\left.W_{t},\left(\mathcal{F}_{t}^{\xi}\right)_{t \in[0, T]}, \xi\right)$ be a weak solution to $(1)$. Let $\mathcal{C}:=C\left([0, T], \mathbb{R}^{d}\right)$ be the space of vector-valued continuous functions. We denote by $\mathcal{B}(\mathcal{C})$ the Borel $\sigma$-field in $\mathcal{C}$ and let $Q:=P^{\xi}$ denote the distribution of $\xi$. Since the solution process $\xi$ can be considered as a random element $\xi: \Omega \rightarrow \mathcal{C}$, the measure space $\left(\mathcal{C}, \mathcal{B}(\mathcal{C}),\left(\Gamma_{t}\right)_{t \in[0, T]}, Q\right)$ appears as its canonical representation (or canonical version), where $\left(\Gamma_{t}\right)$ is a right continuous filtration, $\Gamma_{t}=\beta_{t+}$, $\beta_{t}=\sigma\left\{\pi_{s}: s \in[0, t]\right\}$. Here $\left(\pi_{t}\right)_{t \in[0, T]}$ denotes the canonical projection process, i.e. $\pi_{t}: \mathcal{C} \rightarrow \mathbb{R}^{d}, \pi_{t}(x)=x(t)$ for $x \in \mathcal{C}$, and $t \in[0, T]$. The canonical version of weak solutions allows us to consider them on the same canonical space. In the case of a stochastic single-valued equation, the existence of a weak solution is equivalent to the fact that its distribution is a solution to a local martingale problem (see [13, 14]). A similar approach can be used in the set-valued case.

Let $a:[0, T] \times \mathcal{C} \rightarrow \mathbb{R}^{d}$ and $b:[0, T] \times \mathcal{C} \rightarrow \mathbb{R}^{d \times m}$ be measurable functions. Let $u \in C_{\mathrm{b}}^{2}\left(\mathbb{R}^{d}\right)$, i.e. $u: \mathbb{R}^{d} \rightarrow \mathbb{R}$ is bounded and twice differentiable. Suppose $y \in \mathcal{C}$. We use the following differential operator:

$$
\left(\mathcal{A}_{t} u\right)(y):=\frac{1}{2} \sum_{i=1}^{d} \sum_{k=1}^{d} \gamma_{i k}(t, y) \frac{\partial^{2} u(y(t))}{\partial x_{i} \partial x_{k}}+\sum_{i=1}^{d} a_{i}(t, y) \frac{\partial u(y(t))}{\partial x_{i}},
$$

where $\gamma_{i k}(t, y)=\sum_{j=1}^{m} b_{i j}(t, y) b_{k j}(t, y), 1 \leq i, k \leq d$.

Let $\mathcal{M}(\mathcal{C})$ denote the set of all probability measures on $(\mathcal{C}, \mathcal{B}(\mathcal{C}))$. For given multifunctions $F, G$ and a probability measure $\mu$ on $\left(\mathbb{R}^{d}, \mathcal{B}\left(\mathbb{R}^{d}\right)\right)$ we introduce:

Definition 2. A probability measure $Q \in \mathcal{M}(\mathcal{C})$ is said to be a solution to the local martingale problem for $(F, G, \mu)$ if:

(i) $Q^{\pi_{0}}=\mu$,

(ii) there exist measurable mappings $a:[0, T] \times \mathcal{C} \rightarrow \mathbb{R}^{d}$ and $b:[0, T] \times$ $\mathcal{C} \rightarrow \mathbb{R}^{d \times m}$ such that $a(t, y) \in F(t, y(t)), b(t, y) \in G(t, y(t)) d t \times d Q$-a.e., 
and for every $f \in C_{\mathrm{b}}^{2}\left(\mathbb{R}^{d}\right)$ the process $\left(M_{t}^{f}\right)($ on $(\mathcal{C}, \mathcal{B}(\mathcal{C}), Q))$ defined by

$$
M_{t}^{f}:=f \circ \pi_{t}-f \circ \pi_{0}-\int_{0}^{t}\left(A_{s} f\right) d s, \quad t \in[0, T],
$$

is a $\left(\Gamma_{t}, Q\right)$ - local martingale.

Let $\mathcal{R}^{\text {loc }}(F, G, \mu)$ denote the set of those measures $Q \in \mathcal{M}(\mathcal{C})$ which are solutions to the local martingale problem for $(F, G, \mu)$. The space $\mathcal{M}(\mathcal{C})$, and hence the set $\mathcal{R}^{\operatorname{loc}}(F, G, \mu)$, can be equipped with the topology of weak convergence of probability measures (see e.g. [2]). We denote by $\operatorname{SI}(F, G, \mu)$ the set of weak solutions to the stochastic inclusion (1). Fix an initial distribution $\mu$. We have the following relation between the existence of weak solutions to the stochastic inclusion and solutions to the martingale problem described above.

Theorem 2. Let $F, G:[0, T] \times \mathbb{R}^{d} \rightarrow 2^{\mathbb{R}^{d}}, 2^{\mathbb{R}^{d \times m}}$ be $\mathcal{B}\left([0, T] \times \mathbb{R}^{d}\right)$ measurable multifunctions, and let $\mu$ be a probability measure on $\left(\mathbb{R}^{d}, \mathcal{B}\left(\mathbb{R}^{d}\right)\right)$. Then $\operatorname{SI}(F, G, \mu) \neq \emptyset$ if and only if $\mathcal{R}^{\operatorname{loc}}(F, G, \mu) \neq \emptyset$.

Proof. Let $\left(\Omega, \mathcal{F}, P, W_{t},\left(\mathcal{F}_{t}^{\xi}\right)_{t \in[0, T]}, \xi\right)$ be a weak solution to (1). Then there exist selections $f_{t} \in F\left(t, \xi_{t}\right), g_{t} \in G\left(t, \xi_{t}\right) d t \times d P$-a.e. such that $f_{t}, g_{t}$ are $\mathcal{F}_{t}^{\xi}$-measurable for $t \in[0, T]$. By [10, Lemma 4.9], one can find measurable mappings $a:[0, T] \times \mathcal{C} \rightarrow \mathbb{R}^{d}$ and $b:[0, T] \times \mathcal{C} \rightarrow \mathbb{R}^{d \times m}$ such that $a(t, \cdot)$ and $b(t, \cdot)$ are $\Gamma_{t}$-measurable for $t \in[0, T]$ (hence progressively measurable) such that $a(t, \xi)=f_{t}$ and $b(t, \xi)=g_{t}$. It follows that in the "canonical setting" we have $a(t, y) \in F(t, y(t))$ and $b(t, y) \in G(t, y(t)) d t \times d P^{\xi}$-a.e. Hence, the system $\left(\Omega, \mathcal{F}, P, W_{t},\left(\mathcal{F}_{t}^{\xi}\right)_{t \in[0, T]}, \xi\right)$ is a weak solution to the stochastic differential equation

$$
\begin{aligned}
d \xi_{t} & =a(t, \xi) d t+b(t, \xi) d W_{t}, \\
P^{\xi_{0}} & =\mu .
\end{aligned}
$$

The rest of the proof follows from [5, Proposition 4.11, Ch. 5], which indicates the equivalence between the existence of a weak solution to the stochastic differential equation and the existence of a solution to the local martingale problem for $(a, b, \mu)$, hence for $(F, G, \mu)$. The measures $P$ and $Q$ are related by $Q=P^{\xi}$.

By Theorems 1 and 2 we have

Corollary 1. Under the assumptions of Theorem $1, \mathcal{R}^{\mathrm{loc}}(F, G, \mu) \neq \emptyset$.

Note that the nonemptiness of the set $\mathcal{R}^{\text {loc }}(F, G, \mu)$ can be proved under weaker assumptions on $F$ and $G$.

Proposition. Let $F:[0, T] \times \mathbb{R}^{d} \rightarrow \operatorname{Conv}\left(\mathbb{R}^{d}\right)$ and $G:[0, T] \times \mathbb{R}^{d} \rightarrow$ $\operatorname{Conv}\left(\mathbb{R}^{d \times m}\right)$ be $\mathcal{B}([0, T]) \times \mathcal{B}\left(\mathbb{R}^{d}\right)$-measurable multifunctions such that $F(t, \cdot)$ 
and $G(t, \cdot)$ are lower semicontinuous for $t \in[0, T]$, and suppose that

$$
\max \left\{\|F(t, x)\|^{2},\|G(t, x)\|^{2}\right\} \leq K\left(1+\|x\|^{2}\right) .
$$

Then $\mathcal{R}^{\operatorname{loc}}(F, G, \mu) \neq \emptyset$.

Proof. From [4, Th. 7.23], there exist mappings $f, g:[0, T] \times \mathbb{R}^{d} \rightarrow$ $\mathbb{R}^{d}, \mathbb{R}^{d \times m}$ which are Carathéodory selections for $F$ and $G$ respectively. Let $a:[0, T] \times C \rightarrow \mathbb{R}^{d}$ and $b:[0, T] \times C \rightarrow \mathbb{R}^{d \times m}$ be mappings defined by $a(t, y)=f(t, y(t))$ and $b(t, y)=g(t, y(t))$. Hence they are jointly measurable and continuous with respect to the second variable. Now by [3, Th. 2, Ch. 5 , $\S 2]$ there exists a weak solution to the stochastic equation

$$
\begin{aligned}
d \xi_{t} & =a(t, \xi) d t+b(t, \xi) d W_{t}, \\
P^{\xi_{0}} & =\mu
\end{aligned}
$$

which completes the proof.

3. Topological properties of $\mathcal{R}^{\text {loc }}(F, G, \mu)$. In order to state our optimality result we have to describe the topological properties of the set $\operatorname{SI}(F, G, \mu)$ or equivalently, what we shall do, properties of $\mathcal{R}^{\operatorname{loc}}(F, G, \mu)$. The closedness of $\operatorname{SI}(F, G, \mu)$ has been proved by M. Kisielewicz [8]. In the same paper the compactness of the set of weak solutions was considered only for solutions living on the same filtered probability space. Owing to Theorem 2, a more general result can be proved for the whole set of solutions without the latter restriction. Moreover the result holds true with a weaker l.s.c. assumptions imposed on $F$ and $G$. Namely, we have:

Theorem 3. Let $F:[0, T] \times \mathbb{R}^{d} \rightarrow \operatorname{Conv}\left(\mathbb{R}^{d}\right)$ and $G:[0, T] \times \mathbb{R}^{d} \rightarrow$ $\operatorname{Conv}\left(\mathbb{R}^{d \times m}\right)$ be measurable and bounded set-valued mappings such that $F(t, \cdot)$ and $G(t, \cdot)$ are l.s.c. for each fixed $t \in[0, T]$, and let $\left(\mu^{k}\right)$ be a tight a sequence of probability measures. Then $\bigcup_{k \geq 1} \mathcal{R}^{\operatorname{loc}}\left(F, G, \mu^{k}\right)$ is a nonempty and relatively compact subset of $\mathcal{M}(\mathcal{C})$.

Proof. The nonemptiness follows from Corollary 1. By Prokhorov's Theorem [2], it is enough to show that the set considered is tight. Note first that

$$
\begin{aligned}
\lim _{a \rightarrow \infty} \sup _{Q \in \cup_{k \geq 1}} \operatorname{Rup}_{\mathcal{R}^{\operatorname{loc}}\left(F, G, \mu^{k}\right)} Q\{y \in \mathcal{C}:\|y(0)\|>a\} \\
\quad \leq \lim _{a \rightarrow \infty} \sup _{k \geq 1} \mu^{k}\left\{x \in \mathbb{R}^{d}:\|x\|>a\right\}=0,
\end{aligned}
$$

because the sequence $\left(\mu^{k}\right)$ is tight. Hence by [2, Th. 8.2] it is sufficient to show that for every $\varepsilon>0$,

$$
\lim _{n \rightarrow \infty} \sup _{Q \in \cup_{k \geq 1}} Q \mathcal{R}^{\operatorname{loc}\left(F, G, \mu^{k}\right)}\left\{\left\{y \in C: \Delta_{T}(1 / n, y)>\varepsilon\right\}=0,\right.
$$


where $\Delta_{T}(\delta, y)=\sup \{\|y(t)-y(s)\|: s, t \in[0, T],|s-t|<\delta\}$ for $y \in \mathcal{C}$. Let $Q \in \bigcup_{k \geq 1} \mathcal{R}^{\operatorname{loc}}\left(F, G, \mu^{k}\right)$. Then there exist $k \geq 1$, and measurable and bounded (say by a constant $L>0$ ) mappings $a^{k}, b^{k}:[0, T] \times \mathcal{C} \rightarrow \mathbb{R}^{d}, \mathbb{R}^{d \times m}$ such that $a^{k}(t, y) \in F(t, y(t)), b^{k}(t, y) \in G(t, y(t)) d t \times d Q$-a.e. and $Q \in$ $\mathcal{R}^{\text {loc }}\left(a^{k}, b^{k}, \mu^{k}\right)$. Hence, taking $f: \mathbb{R}^{d} \rightarrow \mathbb{R}, f(x)=x_{i}, i=1, \ldots, d$, we obtain continuous $Q$-local martingales (on $\mathcal{C}$ )

$$
M_{t}^{k, i}=\pi_{t}^{i}-\int_{0}^{t} a_{i}^{k}(s, \cdot) d s,
$$

with quadratic covariations

$$
\left[M^{k, i}, M^{k, j}\right]_{t}=\int_{0}^{t}\left(b^{k}\left(b^{k}\right)^{T}\right)_{i j}(s, \cdot) d s, \quad i, j=1, \ldots, d .
$$

Let $M^{k}=\left(M^{k, 1}, \ldots, M^{k, d}\right)$. For $0 \leq t_{0}<t_{1}<T$, introduce the stopping time $\tau(y)=\inf \left\{u>0:\left\|\pi_{t_{0}+u}(y)-\pi_{t_{0}}(y)\right\|>\varepsilon / 3\right\} \wedge\left(t_{1}-t_{0}\right)$, where $y \in \mathcal{C}$. Then the process $M_{t_{0}+t \wedge \tau}^{k}-M_{t_{0}}^{k}$ is a continuous $\left(\Gamma_{t_{0}+t}, Q\right)$-martingale. We let $t_{0}=0$ for simplicity. Then one can show that

$$
\left\|\pi_{t \wedge \tau}-\pi_{0}\right\|^{2} \leq 2\left\|M_{t \wedge \tau}^{k}-M_{0}^{k}\right\|^{2}+2\left\|\int_{0}^{t \wedge \tau} a^{k}(s, \cdot) d s\right\|^{2} \quad Q \text {-a.e. }
$$

and consequently

$$
\left(\pi-\pi_{0}\right)_{t \wedge \tau}^{* 2} \leq 2\left(M^{k}-M_{0}^{k}\right)_{t \wedge \tau}^{* 2}+2 L^{2} \tau^{2} .
$$

By (5) for any $p \geq 1$ we get

$$
E_{Q}\left(\pi-\pi_{0}\right)_{\tau}^{* 2 p} \leq 2^{p} E_{Q}\left(M^{k}-M_{0}^{k}\right)_{\tau}^{* 2 p}+2^{p} L^{2 p} E_{Q}\left(\tau^{2 p}\right) .
$$

For the continuous $Q$-local martingale $M^{k}-M_{0}^{k}$ we apply Burkholder's inequality (see e.g. [13]) (with the same $p$ ) to get

$$
E_{Q}\left(M^{k}-M_{0}^{k}\right)_{\tau}^{* 2 p} \leq C_{2 p} E_{Q}\left\{\int_{0}^{\tau} \sum_{i=1}^{d}\left(b^{k}\left(b^{k}\right)^{T}\right)_{i i}(s, \cdot) d s\right\}^{p},
$$

where

$$
C_{2 p}=\left\{\left(\frac{2 p-1}{2 p}\right)^{2 p} p(2 p-1)\right\}^{p} .
$$

Thus by the bounds on $F$ and $G$, the inequality (6) has the form

$$
E_{Q}\left(\pi-\pi_{0}\right)_{\tau}^{* 2 p} \leq 2^{p} A L^{p} C_{2 p} E_{Q}\left(\tau^{p}\right)+2^{p} L^{2 p} E_{Q}\left(\tau^{2 p}\right),
$$

where $A$ is some constant depending on $L, p$, and $d$. Restoring $t_{0}$ and setting $\alpha=t_{1}-t_{0}, p=2$, we obtain

$$
E_{Q}\left(\pi-\pi_{t_{0}}\right)_{\alpha}^{* 4} \leq 4 A L^{2} C_{4} \alpha^{2}+4 L^{4} \alpha^{4} .
$$


Hence by the Chebyshev inequality we get, for each $\varepsilon>0$,

$$
Q\left\{\sup _{s \leq \alpha}\left\|\pi_{t_{0}+s}-\pi_{t_{0}}\right\|>\varepsilon\right\} \leq \frac{4 A L^{2} C_{4} \alpha^{2}+4 L^{4} \alpha^{4}}{\varepsilon^{4}} .
$$

Let $T^{*}=[T]+1$. For arbitrary $n \in \mathbb{N}$, divide the interval $\left[0, T^{*}\right]$ by the points $1 / n, i=0,1, \ldots, T^{*} n$. Then

$$
\begin{aligned}
Q\left\{y: \Delta_{T}(1 / n, y)>\varepsilon\right\} & \leq Q\left\{y: \Delta_{T^{*}}(1 / n, y)>\varepsilon\right\} \\
& =Q\left\{\bigcup_{i=0}^{T^{*} n-1}\left\{\sup _{0 \leq s \leq 1 / n}\left\|\pi_{i / n+s}-\pi_{i / n}\right\|>\varepsilon / 3\right\}\right\} .
\end{aligned}
$$

Hence using (7) with $\alpha=1 / n$, we get

$$
Q\{y: \Delta(1 / n, y)>\varepsilon\} \leq 3^{4} T^{*}\left(\frac{4 A L^{2} C_{4}}{n \varepsilon^{4}}+\frac{4 L^{4}}{n^{3} \varepsilon^{4}}\right),
$$

which proves (4) and completes the proof.

Corollary 2. If $F:[0, T] \times \mathbb{R}^{d} \rightarrow \operatorname{Conv}\left(\mathbb{R}^{d}\right)$ and $G:[0, T] \times \mathbb{R}^{d} \rightarrow$ $\operatorname{Conv}\left(\mathbb{R}^{d \times m}\right)$ are measurable bounded set-valued mappings such that $F(t, \cdot)$ and $G(t, \cdot)$ are l.s.c. for each fixed $t \in[0, T]$, then for every probability measure $\mu$ on $\left(\mathbb{R}^{d}, \mathcal{B}\left(\mathbb{R}^{d}\right)\right)$ the set $\mathcal{R}^{\text {loc }}(F, G, \mu)$ is nonempty and relatively compact in $\mathcal{M}(\mathcal{C})$.

The closedness of the set of weak solutions is another important property. Let us recall the following definition.

Definition 3 ([8]). A set $U \subset \mathbb{R}^{d \times m}$ is said to be diagonally convex if the set $D(U)=\left\{u u^{T}: u \in U\right\}$ is a convex subset of $\mathbb{R}^{d \times d}$.

It can be shown (see [8]) that if $U \subset \mathbb{R}^{1 \times m}$ is convex then it is also diagonally convex. A set-valued mapping $G:[0, T] \times \mathbb{R}^{d} \rightarrow \operatorname{Conv}\left(\mathbb{R}^{d \times m}\right)$ will be called diagonally convex valued if $G(t, x)$ is diagonally convex for every $t \in[0, T]$ and $x \in \mathbb{R}^{d}$. In [8] the following result was proved.

Theorem 4 (Kisielewicz [8]). Let $F:[0, T] \times \mathbb{R}^{d} \rightarrow \operatorname{Conv}\left(\mathbb{R}^{d}\right)$ and $G:$ $[0, T] \times \mathbb{R}^{d} \rightarrow \operatorname{Conv}\left(\mathbb{R}^{d \times m}\right)$ be measurable bounded set-valued mappings such that $F(t, \cdot)$ and $G(t, \cdot)$ are continuous for each fixed $t \in[0, T]$. Assume also that $G$ is diagonally convex valued. Then, for every probability measure $\mu$ on $\left(\mathbb{R}^{d}, \mathcal{B}\left(\mathbb{R}^{d}\right)\right)$ the set $\operatorname{SI}(F, G, \mu)$ is nonempty and closed with respect to convergence in distribution.

Using the equivalent martingale problem approach, by Theorems 3 and 4 , we can state:

Corollary 3. Let $F:[0, T] \times \mathbb{R}^{d} \rightarrow \operatorname{Conv}\left(\mathbb{R}^{d}\right)$ and $G:[0, T] \times \mathbb{R}^{d} \rightarrow$ $\operatorname{Conv}\left(\mathbb{R}^{d \times m}\right)$ be measurable bounded set-valued mappings such that $F(t, \cdot)$ 
and $G(t, \cdot)$ are continuous for each fixed $t \in[0, T]$. Assume also that $G$ is diagonally convex valued. Then, for every probability measure $\mu$ on $\left(\mathbb{R}^{d}, \mathcal{B}\left(\mathbb{R}^{d}\right)\right)$, the set $\mathcal{R}^{\text {loc }}(F, G, \mu)$ is nonempty and compact in $\mathcal{M}(\mathcal{C})$.

To study the dependence of $\mathcal{R}^{\text {loc }}(F, G, \mu)$ on the initial distribution, we recall the following characterization of upper semicontinuity of set-valued mappings. Below, Comp() denotes the space of all nonempty compact subsets of the underlying space.

Theorem 5 ([6]). Let $X$ and $Y$ be metric spaces. A set-valued mapping $Z: X \rightarrow \operatorname{Comp}(Y)$ is u.s.c. if and only if for every $x \in X$ and every sequence $x_{n} \rightarrow x$, each sequence $y_{n} \in Z\left(x_{n}\right)$ has a subsequence converging to a limit belonging to $Z(x)$.

Let $\mathcal{M}\left(\mathbb{R}^{d}\right)$ denote the space of probability measures on $\left(\mathbb{R}^{d}, \mathcal{B}\left(\mathbb{R}^{d}\right)\right)$ equipped with the topology of weak convergence. By the corollary above we can introduce the solution set mapping $\mathcal{R}^{\text {loc }}(F, G, \cdot): \mathcal{M}\left(\mathbb{R}^{d}\right) \rightarrow$ $\operatorname{Comp}(\mathcal{M}(\mathcal{C}))$. Since the topology of weak convergence of probability measures both in $\mathcal{M}\left(\mathbb{R}^{d}\right)$ and $\mathcal{M}(\mathcal{C})$ can be metrized by Prokhorov's metrics (see e.g. [2]), under the assumptions of Corollary 3 the mapping $\mu \mapsto$ $\mathcal{R}^{\text {loc }}(F, G, \mu)$ can be considered as a multifunction defined on the metric space $\mathcal{M}\left(\mathbb{R}^{d}\right)$ with values in $\operatorname{Comp}(\mathcal{M}(\mathcal{C}))$. Let $\mu$ be an arbitrary initial distribution and let $\left(\mu_{k}\right)$ be a sequence of probability measures weakly converging to $\mu$. By Theorem 3 , the set $\bigcup_{k \geq 1} \mathcal{R}^{\text {loc }}\left(F, G, \mu^{k}\right)$ is relatively compact. This implies that any sequence $\left(Q^{k}\right)$ such that $Q^{k} \in \mathcal{R}^{\operatorname{loc}}\left(F, G, \mu^{k}\right)$, has a subsequence converging to some probability measure $Q^{\sim} \in \mathcal{M}(\mathcal{C})$. By the Continuous Mapping Theorem (see e.g. [2]) it follows that $\left(Q^{\sim}\right)^{\pi_{0}}=\mu$. As in $\left[8\right.$, Th. 12] it can be shown that $Q^{\sim} \in \mathcal{R}^{\text {loc }}(F, G, \mu)$. By Theorem 5 we have:

THEOREM 6. Under the assumptions of Corollary 3, the mapping $\mu \mapsto$ $\mathcal{R}^{\operatorname{loc}}(F, G, \mu)$ is a u.s.c. multifunction from $\mathcal{M}\left(\mathbb{R}^{d}\right)$ into $\operatorname{Comp}(\mathcal{M}(\mathcal{C}))$.

REMARK 2. The basic consequence of the above theorem is the measurable dependence of the solution set on the initial distribution in the sense of measurability of set-valued mappings (see e.g. [4], [6]).

Using Proposition 2.3 of [6] we obtain an immediate consequence of this theorem, which can be viewed as a set-valued version of Levakov's result on compactness of the weak solution set for the stochastic inclusion (2) (see [9, Th. 2]).

Corollary 4. Let $\Pi$ be a compact subset of $\mathcal{M}\left(\mathbb{R}^{d}\right)$. Then under the assumptions of Corollary 3 , the set $\mathcal{R}^{\mathrm{loc}}(F, G, \Pi):=\bigcup_{\mu \in \Pi} \mathcal{R}^{\mathrm{loc}}(F, G, \mu)$ is a compact subset of $\mathcal{M}(\mathcal{C})$. 
4. Optimal solutions to stochastic inclusions. First, observe that for each weak solution $\left(\Omega, \mathcal{F}, P, W_{t},\left(\mathcal{F}_{t}^{\xi}\right)_{t \in[0, T]}, \xi\right)$ and an arbitrary integrable function $h: \mathbb{R}_{+} \times \mathbb{R}^{d} \rightarrow \mathbb{R}$ we have

$$
E_{P}\left[\int_{0}^{T} h\left(t, \xi_{t}\right) d t\right]=E_{Q}\left[\int_{0}^{T} h\left(t, \pi_{t}\right) d t\right]
$$

and for this reason, the optimality problem described by (3) in the Introduction can be rewritten as

$$
E_{Q^{*}}\left[\int_{0}^{T} h\left(t, \pi_{t}\right) d t\right]=\sup _{Q} E_{Q}\left[\int_{0}^{T} h\left(t, \pi_{t}\right) d t\right],
$$

where the supremum is taken over the set of all distributions of weak solutions to (1) and $Q^{*}$ is the distribution of an optimal weak solution $\left(\Omega^{*}, \mathcal{F}^{*}, P^{*}, W_{t}^{*},\left(\mathcal{F}_{t}^{\xi^{*}}\right)_{t \in[0, T]}, \xi^{*}\right)$.

Hence we introduce the following definition.

Definition 4. By an optimal solution to the inclusion (1) we mean a probability measure $Q^{*} \in \mathcal{R}^{\operatorname{loc}}(F, G, \mu)$ such that

$$
E_{Q^{*}}\left[\int_{0}^{T} h\left(t, \pi_{t}\right) d t\right]=\sup _{Q \in \mathcal{R}^{\operatorname{loc}}(F, G, \mu)} E_{Q}\left[\int_{0}^{T} h\left(t, \pi_{t}\right) d t\right] .
$$

REMARK 3. It is clear that the optimal solution can depend on the initial distribution $\mu$. In fact it will be shown (see Theorem 7 below) that it depends on it in a measurable way.

The proof of the next lemma is standard so it is omitted here.

Lemma. Let $U: \mathcal{M}(\mathcal{C}) \rightarrow \mathbb{R}$ be a continuous mapping. Then the mappings $S: \operatorname{Comp}(\mathcal{M}(\mathcal{C})) \ni K \mapsto \sup _{Q \in K} U(Q) \in \mathbb{R}$ and $W: \operatorname{Comp}(\mathcal{M}(\mathcal{C})) \ni$ $K \mapsto\{Q \in K: U(Q)=S(K)\} \in \operatorname{Comp}(\mathcal{M}(\mathcal{C}))$ are measurable.

We have the following optimality result.

Theorem 7. Let $h:[0, T] \times \mathbb{R}^{d} \rightarrow \mathbb{R}$ be a bounded continuous function. Then under the assumptions of Corollary 3 , there exists a measurable mapping $Q^{*}: \mathcal{M}\left(\mathbb{R}^{d}\right) \rightarrow \mathcal{M}(\mathcal{C})$ such that:

(a) $Q_{\mu}^{*} \in \mathcal{R}^{\text {loc }}(F, G, \mu)$ for every $\mu \in \mathcal{M}\left(\mathbb{R}^{d}\right)$,

(b) $Q_{\mu}^{*}$ is an optimal solution for every $\mu \in \mathcal{M}\left(\mathbb{R}^{d}\right)$.

Proof. We have to prove the existence of a measurable mapping $Q_{\mu}^{*} \in$ $\mathcal{R}^{\text {loc }}(F, G, \mu)$ such that 


$$
E_{Q_{\mu}^{*}}\left[\int_{0}^{T} h\left(t, \pi_{t}(y)\right) d t\right]=\sup _{Q \in \mathcal{R}^{\operatorname{loc}}(F, G, \mu)} E_{Q}\left[\int_{0}^{T} h\left(t, \pi_{t}(y)\right) d t\right]
$$

for every $\mu \in \mathcal{M}\left(\mathbb{R}^{d}\right)$. Recall once again that by Theorem $6, \mathcal{R}^{\text {loc }}(F, G, \cdot)$ is a measurable set-valued mapping. Define

$$
\begin{aligned}
\mathcal{R}^{\mathrm{loc}}(F, G, \mu)^{*}= & \left\{Q \in \mathcal{R}^{\mathrm{loc}}(F, G, \mu):\right. \\
& \left.E_{Q}\left[\int_{0}^{T} h\left(t, \pi_{t}(y)\right) d t\right]=\sup _{Q \in \mathcal{R}^{\operatorname{loc}}(F, G, \mu)} E_{Q}\left[\int_{0}^{T} h\left(t, \pi_{t}(y)\right) d t\right]\right\} .
\end{aligned}
$$

Since the function $\mathcal{C} \ni y \mapsto \int_{0}^{T} h\left(t, \pi_{t}(y)\right) d t \in \mathbb{R}$ is continuous and bounded, the mapping $U(Q)=E_{Q}\left[\int_{0}^{T} h\left(t, \pi_{t}(y)\right) d t\right]$ is continuous as well. Thus $\mathcal{R}^{\text {loc }}(F, G, \mu)^{*} \in \operatorname{Comp}(\mathcal{M}(\mathcal{C}))$ for every $\mu \in \mathcal{M}\left(\mathbb{R}^{d}\right)$. In the notation of the Lemma, $\mathcal{R}^{\text {loc }}(F, G, \cdot)^{*}=W \circ \mathcal{R}^{\text {loc }}(F, G, \cdot)$, which implies the measurability of $\mathcal{R}^{\text {loc }}(F, G, \cdot)^{*}$. Finally applying the Kuratowski and Ryll-Nardzewski Measurable Selection Theorem (see e.g. [6]) we get the desired measurable measure-valued mapping $Q^{*}$.

REMARK 4. Note that the proof above shows in particular that under the assumptions of Theorem 7 , the set $\mathcal{R}^{\operatorname{loc}}(F, G, \mu)^{*}$ of optimal solutions to $(1)$ is a measurable and compact valued mapping.

Finally, we consider so called weakly viable solutions to the stochastic inclusion (1).

For a fixed, nonempty and closed set $K \subset \mathbb{R}^{d}$ and $\varepsilon \in[0,1)$, define $\Pi(K, \varepsilon)=\left\{\mu \in \mathcal{M}\left(\mathbb{R}^{d}\right): \mu K \geq 1-\varepsilon\right\}$. We say that the stochastic inclusion (1) has a weakly viable (or $\varepsilon$-viable) solution in $K$ if for every $\mu \in \Pi(K, \varepsilon)$, there exists a weak solution $\left(\Omega, \mathcal{F}, P, W_{t},\left(\mathcal{F}_{t}^{\xi}\right)_{t \in[0, T]}, \xi\right)$ of $(1)$ such that $P^{\xi_{t}} \in \Pi(K, \varepsilon), t \in[0, T]$. Recall that for strong solutions the viability problem for $\varepsilon=0$ was studied first by Aubin and Da Prato (see e.g. [1]). Weakly viable solutions to controlled diffusion equations were considered in [11]. By [2, Th. 2.1], it is easy to see that $\Pi(K, \varepsilon)$ is a closed subset of $\mathcal{M}\left(\mathbb{R}^{d}\right)$. Similarly it can be shown that the mapping $Q \mapsto Q^{\pi_{t}}\{K\}$ is u.s.c., for every $t \in[0, T]$. Thus, $Q \mapsto \inf _{t \in[0, T]} Q^{\pi_{t}}\{K\}$ is u.s.c. as well. Consequently, $\mathcal{D}:=\left\{Q \in \mathcal{M}(\mathcal{C}): \inf _{t \in[0, T]} Q^{\pi_{t}}\{K\} \geq 1-\varepsilon\right\}$ is a closed subset of $\mathcal{M}(\mathcal{C})$.

Let $\mu \in \Pi(K, \varepsilon)$ and suppose that the assumptions of Corollary 3 are satisfied. If $\mathcal{R}^{\text {loc }}(F, G, \mu) \cap \mathcal{D} \neq \emptyset$, then by Theorem 6 and the properties of upper semicontinuous set-valued mappings (see e.g. [4, 6]), the map- 
ping $\mathcal{R}_{K, \varepsilon}^{\text {loc }}(F, G, \cdot): \Pi(K, \varepsilon) \rightarrow \operatorname{Comp}(\mathcal{M}(\mathcal{C}))$ defined by $\mathcal{R}_{K, \varepsilon}^{\text {loc }}(F, G, \cdot)=$ $\mathcal{R}^{\text {loc }}(F, G, \cdot) \cap \mathcal{D}$ is u.s.c. as well. Thus, as in the proof of Theorem 7 one can show:

ThEOREM 8. Under the assumptions of Theorem 7, if $\mathcal{R}^{\mathrm{loc}}(F, G, \mu)$ $\cap \mathcal{D} \neq \emptyset$ for $\mu \in \Pi(K, \varepsilon)$, then:

(i) the set-valued mapping $\mathcal{R}_{K, \varepsilon}^{\text {loc }}(F, G, \cdot): \Pi(K, \varepsilon) \rightarrow \operatorname{Comp}(\mathcal{M}(\mathcal{C}))$ is u.s.c.,

(ii) there exists a measurable mapping $Q^{+}: \Pi(K, \varepsilon) \rightarrow \mathcal{M}(C)$ such that $Q_{\mu}^{+} \in \mathcal{R}_{K, \varepsilon}^{\text {loc }}(F, G, \mu)$ and

$$
E_{Q_{\mu}^{+}}\left[\int_{0}^{T} h\left(t, \pi_{t}\right) d t\right]=\sup _{Q \in \mathcal{R}_{K, \varepsilon}^{\operatorname{loc}}(F, G, \mu)} E_{Q}\left[\int_{0}^{T} h\left(t, \pi_{t}\right) d t\right]
$$

for every $\mu \in \Pi(K, \varepsilon)$.

REmark 5. The condition $\mathcal{R}^{\operatorname{loc}}(F, G, \mu) \cap \mathcal{D} \neq \emptyset$ is fulfilled under the so called weak tangential condition (see $[11,12]$ and simply means that there exists a weakly viable solution to (1). Part (ii) of Theorem 8 indicates the existence of a weakly viable optimal solution to this inclusion.

\section{References}

[1] J. P. Aubin and G. Da Prato, The viability theorem for stochastic differential inclusions, Stochastic Anal. Appl. 16 (1998), 1-15.

[2] P. Billingsley, Convergence of Probability Measures, Wiley, New York, 1968.

[3] I. I. Gikhman and A. V. Skorokhod, Stochastic Differential Equations and Their Applications, Kiev, 1982 (in Russian).

[4] S. Hu and N. Papageorgiou, Handbook of Multivalued Analysis, Vol. 1, Theory, Kluwer, Boston, 1997.

[5] I. Karatzas and S. Shreve, Brownian Motion and Stochastic Calculus, Springer, 1988.

[6] M. Kisielewicz, Differential Inclusions and Optimal Control, Kluwer, Dordrecht, 1991.

[7] -, Set-valued stochastic integrals and stochastic inclusions, Stochastic Anal. Appl. 16 (1997), 783-800.

[8] - Weak compactness of solution sets to stochastic differential inclusions with convex right-hand sides, Topol. Methods Nonlinear Anal. 18 (2001), 149-171.

[9] A. A. Levakov, Stochastic differential inclusions, J. Differential Equations 33 (1997), $212-221$.

[10] R. Liptser and A. Shiryaev, Statistics of Stochastic Processes, PWN, 1981 (in Polish).

[11] L. Mazliak, A note on weak viability for controlled diffusion, Statist. Probab. Lett. 49 (2000), 331-336. 
[12] M. Michta, Weak solutions to stochastic differential inclusions. A martingale approach, in: Proc. 3-rd Polish Sympos. Nonlinear Analysis, Lecture Notes Nonlinear Anal. 3, 2002, 141-149.

[13] C. Rogers and D. Williams, Diffusions, Markov Processes, and Martingales, Vol. II, Wiley, 1990.

[14] D. Stroock and S. R. Varadhan, Multidimensional Diffusion Processes, Springer, 1975 .

Institute of Mathematics

University of Zielona Góra

Podgórna 50

65-246 Zielona Góra, Poland

E-mail: m.michta@im.uz.zgora.pl

Received on 25.9.2001;

revised version on 12.7.2002 